\title{
DREAMs make plant cells to cycle or to become quiescent
}

\author{
Zoltán Magyar¹, László Bögre² and Masaki Ito ${ }^{3,4}$
}

\author{
Addresses \\ ${ }^{1}$ Institute of Plant Biology, Biological Research Centre, Szeged, Hungary \\ ${ }^{2}$ Royal Holloway, University of London, School of Biological Sciences, Egham, Surrey, \\ TW20 0EX, UK \\ ${ }^{3}$ Graduate School of Bioagricultural Sciences, Nagoya University, Chikusa, Nagoya \\ 464-8601, Japan \\ ${ }^{4}$ JST, CREST, Chikusa, Nagoya 464-8601, Japan
}

Corresponding author: Ito, Masaki (masakito@agr.nagoya-u.ac.jp)

\begin{abstract}
Cell cycle phase specific oscillation of gene transcription has long been recognized as an underlying principle for ordered processes during cell proliferation. The G1/S- and G2/M-specific cohorts of genes in plants are regulated by the E2F and the MYB3R transcription factors. Mutant analysis suggests that activator E2F functions might not be fully required for cell cycle entry. In contrast, the two activator-type MYB3Rs are part of positive feedback loops to drive the burst of mitotic gene expression, which is necessary at least to accomplish cytokinesis. Repressor MYB3Rs act outside the mitotic time window during cell cycle progression, and are important for the shutdown of mitotic genes to impose quiescence in mature organs. The two distinct classes of E2Fs and MYB3Rs together with the RETINOBLATOMA RELATED are part of multiprotein complexes that may be evolutionary related to what is known as DREAM complex in animals. In plants, there are multiple such complexes with distinct compositions and functions that may be involved in the coordinated cell cycle and developmental regulation of E2F targets and mitotic genes.
\end{abstract} http://www.elsevier.com/open-access/userlicense/1.0/ 


\section{Introduction}

Plant growth and development are reliant on maintaining cell proliferation within meristematic zones, regulated by the perpetual transitions through cell cycle phases and the decision to enter into quiescence accompanied by cellular differentiation programs. The sequence of events through the cell cycle is underpinned by the oscillatory transcription of phase-specific genes, such as the cohort of early, G1/S-specific cell cycle genes, required for the entry into and progression through DNA synthesis, and late, G2/M-specific cell cycle genes with mitotic functions [1]. Gene expression profiling during synchronized cell cycle demonstrated that a significant proportion of genes, around thousand, are cell cycle regulated in yeast (416 genes) [2], human (731 genes) [3] and plant cells (1082 genes in Arabidopsis) [4,5]. How these waves of transcription are connected is best understood in yeast, where the transcription factors themselves are periodically transcribed during the cell cycle and function to activate oncoming and inhibit previous regulators in a fully connected regulatory circuit [6]. However, the identity of the transcription factors and their regulation in metazoans and plants are distinct from that in yeast $[7,8]$. In animal and plant cells, the expression of early cell cycle genes are thought to be induced by the activator-type E2F family transcription factors [8,9], but it is becoming apparent that these E2Fs are not required to drive cell proliferation $[10,11]$. In plants, the late cell cycle genes are regulated by R1R2R3-Myb (MYB3R) family transcription factors [12]. When cells exit cell proliferation, both the G1/S and G2/M cohorts of cell cycle genes must be repressed to allow differentiation programs to commence. The timing of this program is a key determinant of organ size [13]. A fundamental question is whether the developmental down-regulation of cell cycle genes is due to active repression, as cells become quiescent, or the consequence of the lack of transcriptional activation. Recent studies provided clear evidence that the active repression of late cell cycle genes is mediated by the repressor-type MYB3Rs in differentiated cells and when this is abrogated, there is hyperplasia and larger organs; including seeds, roots and leaves [14]. The evolutionary conserved Retinoblastoma (Rb) protein, called RETINOBLASTOMA RELATED (RBR) in plants is another repressor that when silenced, the quiescence of plant cells is broken and cells over-proliferate $[15,16]$.

Within the animal kingdom, the E2F, Myb and Rb-related proteins are found to be part of a conserved protein complex, called DP, Rb-like, E2F, and MuvB (DREAM) in 
human, Drosophila RBF, E2f2 and Mip complex (dREAM) in fruit fly and DP, RB and MuvB complex (DRM) in nematode [17-20] (for further details on DREAM complexes in different organisms; see Box 1). DREAM complexes have important roles in repressing cell cycle genes during quiescence and in the co-ordination of their phase-specific expression [17]. A recent work in Arabidopsis showed the participation of MYB3Rs, E2Fs and RBR in a common complex, suggesting that DREAM complexes are evolutionarily conserved between kingdoms [14,21]. Although the regulation of cell cycle genes by MYB3Rs and E2Fs have been studied and argued separately [8], the discovery of the large protein complexes containing both transcription factors give rise to the possibility of coordinated regulation of the early and late cell cycle genes during and as cells exit the cell cycle [22].

\section{Activator E2F transcription factors might not be essential for cell cycle entry}

The E2F transcription factors are considered to be the main regulators of early cell cycle gene transcription, but it is becoming apparent that they have much broader targets, including mitotic and cell cycle independent functions [10]. E2Fs were classified as repressors that in association with RBR impose quiescence and activators that when released upon RBR phosphorylation drives the entry into proliferation by transcriptional activation of cell cycle genes [23]. Arabidopsis holds three different E2Fs, nominated E2FA, E2FB and E2FC. E2FC is considered to be a repressor, because its overexpression down-regulated the replication initiation gene, $C D C 6$, while its silencing increased CDC6 expression and led to ectopic cell proliferation [24,25]. E2FC silencing effects are reminiscent to RBR co-suppression phenotypes, supporting the notion that these two proteins act together in a repressor complex [26]. E2FA expression peaks during S-phase and when overexpressed it induced the expression of S-phase genes [27]. This would indicate that E2FA is an activator, but studies with the overexpression of a C-terminally truncated mutant E2FA form that lost the abilities both for transactivation and for binding to RBR, yet is fully capable to drive the expression of S-phase genes and to induce endoreduplication [28].These experiments rather suggest that the release of E2FA from RBR repression is required for these functions. E2FB is uniformly expressed throughout the cell cycle, and when overexpressed it activates the expression of cell cycle genes, including the mitotic CDKB1;1 and it leads to sustained cell proliferation even in the absence of auxin in cultured tobacco cells, suggesting an 
activator function [29]. However, mutants in E2F genes individually, or in combinations, such as the $e 2 f a / b$ double [30] or $e 2 f a / b / c$ triple [11] mutants are viable and only compromised in cell cycle-unrelated functions, such as resistance to pathogens [11]. Thus, in accordance to fly and mouse studies [23,31], activator E2Fs are not universally required for cell proliferation. It is becoming apparent that E2Fs in association with RBR are important as repressors to maintain meristem functions, ensuring cell survival and imposing quiescence during cell cycle exit. RBR and E2Fs also have cell cycle independent functions.

\section{MYB3Rs shape the wave of G2/M-specific transcription}

In Arabidopsis, approximately 180 late cell cycle genes are coordinately expressed during late G2 and M phases [32]. The transcription of this cohort of mitotic genes is regulated by the cis-acting MITOSIS-SPECIFIC ACTIVATOR (MSA) element in their promoters, where MYB3R transcription factors bind [12,33,34]. The Arabidopsis genome contains five genes encoding MYB3R proteins. MYB3R1 and MYB3R4 are closely related, the former is constitutive, while the latter is mitotically expressed [35]. The combined mutations in these two genes diminished the expression of the late cell cycle genes, suggesting that these two MYB3Rs are redundantly required for their transcriptional activation $[32,35]$. Consistent with the regulation of mitotic functions is the frequent occurrence of incomplete cytokinesis in the myb3r1/4 double mutant that is largely due to the reduced expression of one of the targets, KNOLLE, involved in cell plate formation [35]. In tobacco cell culture, the mitotic MYB3R was shown to be activated through direct phosphorylation by CDKA in association with a mitotic cyclin [35, 36]. Mitotic cyclins are themselves transcriptionally activated by the activator MYB3R [36], thus constituting a positive feedback loop. The mitotically expressed MYB3R is also autoregulated through the MSA motifs in its own promoter [37]. These two amplifying mechanisms for the accumulation of activator MYB3Rs in turn triggers the burst of expression of downstream targets required for the entry and progression through mitosis (Figure 1).

In contrast to MYB3R1 and MYB3R4, the other pair of highly similar MYB3R3 and MYB3R5, when mutated together, led to the upregulation of mitotic genes both in proliferating and in non-proliferating quiescent cells, suggesting repressive functions [14]. During the cell cycle progression, these repressive MYB3Rs may operate as a 
mask to reduce the basal expression of mitotic genes outside their normal time window and thus to reinforce the periodic expression of these genes [14] (Figure 1). Surprisingly, MYB3R1 can synergize with MYB3R4 as an activator, while together with MYB3R3 and MYB3R5 it acts as a repressor [14]. This may suggest that MYB3R1 is part of the switch that determines repressor activity outside the G2/M phase while at the G2/M transition, when CDK activity rises, MYB3R1 may become activated for transactivation by CDK phosphorylation, leading to the early induction of mitotic genes (Figure 1). This initial switch acting on MYB3R1 might get further amplified by the mitotically expressed MYB3R4 to trigger the full G2/M transition through the burst of late cell cycle gene expression.

\section{Cell proliferation and differentiation, mutual exclusion?}

Cell cycle genes are normally repressed in mature organs. For mitotic genes, this developmental repression is dependent on the repressor MYB3Rs [14]. This was demonstrated by the widespread occurrence of the CYCB1;2 reporter in myb3r1/3/5 triple mutant even in terminally differentiated cells, such as trichomes and root hair cells [14]. This suggests that in plants an active repression of mitotic transcription operates not only in developmentally quiescent, but even in fully differentiated cells. Upregulation of CYCB1;1 was also observed when the E2FC transcription factor is silenced [25], but this needs to be confirmed in stable knockout mutant lines. Silencing of RBR delays [26], while overexpression instigate differentiation programs [38], suggesting that RBR is a key determinant for this transition. Therefore, RBR, E2Fs and MYB3Rs have similar roles in cell cycle exit and shared function in the repression of cell cycle genes as cells differentiate. This is consistent with the finding that they are part of multiple repressor complexes (see below).

RBR also forms a repressor complex with E2FA, but this happens in proliferating cells within the meristem [28]. The RBR-E2FA complex is not disrupted by elevated CYCD3;1 levels leading to increased RBR phosphorylation. It was suggested that the function of this RBR-E2FA complex is to repress differentiation genes, such as expansins and the endoreduplication-inducing CCS52A for the maintenance of cell proliferation in the meristems [28]. Whether the RBR-E2FA is part of a repressor complex is not yet known. 


\section{DREAM complexes with conserved and species-specific attributes}

Using chromatin immunoprecipitation genome wide (ChIP-seq), it was shown that the repressor MYB3R3 not only associates with promoters of mitotic genes, but also with the early cell cycle genes that were previously defined as E2F targets [14]. Because these early cell cycle genes show significant enrichment for E2F motifs, but not for MSA elements in their promoter regions, the DNA binding is not through MYB3R3. In agreement, co-immunoprecipitation experiments showed the association of MYB3R3 with E2FC and RBR [14], that evoked the existence of larger protein complexes known to bring together these two classes of transcription factors and Rb for the repression of cell cycle genes, called dREAM in fly [39]. Similar protein complexes were found in human and nematode, suggesting evolutionary conserved assemblies and functions [17] (Table 1, Box1). Searches for Arabidopsis genes orthologous to the core components identified ALWAYS EARLY (ALY) genes (orthologous to LIN9/Mip130), genes coding for the Tesmin/TSO1-like CXC domain-containing (TCX) proteins (orthologous to LIN54/Mip120) and the MULTICOPY SUPRESSOR OF IRA1 (MSI1) [14] (Table 1). MSI1 is a histone binding protein that was shown to co-purify with HISTONE DEACETYLASE19 (HDA19) [40], and MSI1 together with RBR were found to be required for epigenetic regulation of imprinted genes [41]. However, there are no plant orthologues for the other core components, LIN37/Mip40 and LIN52 (Table 1). Thus, there is a convergence on some common DREAM complexes components in evolutionary divergent species, but there are important differences how these complexes assemble and function. In human cells, the common core complex (called MuvB core) either associates with E2F4/5 to impose G1 arrest or with the B-Myb or the mitotic forkhead class transcription factors, FoxM1 that function to induce the waves of S- and G2/M-specific gene expression [42]. In Arabidopsis, it appears that both the activatorand repressor-type DREAM-like complexes contain RBR, but in meristematic cells, E2FB and the mitosis-specific MYB3R4 is recruited to activate late cell cycle genes, while in mature leaves the repressor MYB3R3 and E2FC are present (Table 1, Figure 2).

\section{The Arabidopsis DREAMs of cell cycle control}

The existence of repressor- and activator-types of Myb transcription factors and their co-recruitment with the repressor E2FC or activator E2FB to RBR-containing 
DREAM-like complexes is a plant-specific utilization of these transcriptional regulatory module. How these divergent RBR containing DREAM-like complexes attain activator or repressor functions, how their dynamic interchange is regulated are largely unresolved questions. CDKA;1 was found to be developmental-specifically recruited to both MYB3R4 and MYB3R3 containing DREAM-like complexes [14]. RBR is an ancient signal-dependent scaffold for protein interactions, regulated by multisite phosphorylations governed by CDKs. These phosphorylation events not necessarily leading to the disassembly of the complex, as was found in Chlamydomonas [43]. In animal cells, it was shown that Rb phosphorylation at distinct sites can diversify RBR complexes towards different functions, e.g. to become activator on certain batteries of genes while repressor on others [44]. Such diversification of RBR functions upon phosphorylation may also exist in plants, as suggested by the distinct association of E2FA and E2FB to RBR upon phosphorylation by CYCD3;1 dependent CDK, induced by sucrose [28]. RBR can also be targeted by phosphorylation through other signalling pathways, such as the stress-induced mitogen activated protein kinase [45], MPK6 that when mutated leads to hyperplasia, larger seeds, roots [46]. The phosphorylation status of RBR, a common component of multiple DREAM-like complexes in Arabidopsis, might determine the biochemical properties of the complex as well as its assembly and disruption.

\section{Concluding remarks}

Plant-specific properties of the multiprotein complex containing both E2F and MYB3R may provide new insights for various unsolved phenomena unique to plants. In plants, even the fully differentiated somatic and generative cells can be readily reprogramed to become undifferentiated and capable of proliferating to allow organ regeneration or somatic embryogenesis [47]. A central part of this regeneration program is the de-repression of cell cycle genes, which may be mediated by the DREAM-like complex in plants. Plants may employ the mechanisms different from animals for establishing and maintaining the repressed state for cell cycle genes, which are more readily de-repressible upon stimuli. Future study should be focused on the molecular basis of post-mitotic repression mediated by DREAM-like complexes that may explain the extraordinarily high plasticity of plant cells.

Endoreduplication is integral to a number of plant developmental programs, which 
relies on the induction of early but repression of late cell cycle genes. This coordinated regulation of the cell cycle genes may require protein complexes where MYB3R and E2F may functionally interact with each other. It is known that E2F2 acts in opposition to Myb to repress some of the late cell cycle genes in fly dREAM complex [48]. Endoreduplication is also known to occur during pathogen infections in plants [49]. For instance, it was reported that the mitotic MYB3R4 has an essential involvement in the induction of endoreduplication at the infection sites of the obligate biotrophic fungus, powdery mildew [50], suggesting that a DREAM-like complex may be integral for this cellular response caused by pathogens. Thus, there are accumulating evidence to suggest broader involvements of cell cycle regulators in plant responses to pathogens as well as to environmental factors [51, 52]. The RBR-MYB3R-E2F complexes may provide convergence points to signaling mechanisms in biotic interaction, abiotic stresses, and development to coordinately regulate the expression of early and late cell cycle genes, thereby modulating cell proliferation, endoreduplication, or quiescence. Future research will have to address how these divergent DREAM complexes become hubs to regulate plant growth and development in tune with environmental conditions.

\section{Acknowledgements}

The research in the authors' laboratory on the topic of this review is supported by the grant from JSPS KAKENHI (grant numbers 26291058, 26660290, and 16H01236), and JST, CREST to M.I, by the BBSRC-NSF grant (BB/M025047/1) to L.B. and by the Hungarian Academy, OTKA 105816 to Z.M.

\section{References and recommended reading}

1. Breeden LL: Periodic transcription: a cycle within a cycle. Curr Biol 2003, 13:R31-38.

2. Cho RJ, Campbell MJ, Winzeler EA, Steinmetz L, Conway A, Wodicka L, Wolfsberg TG, Gabrielian AE, Landsman D, Lockhart DJ, et al.: A genome-wide transcriptional analysis of the mitotic cell cycle. Mol Cell 1998, 2:65-73.

3. Cho RJ, Huang M, Campbell MJ, Dong H, Steinmetz L, Sapinoso L, Hampton G, Elledge SJ, Davis RW, Lockhart DJ: Transcriptional regulation and function during the human cell cycle. Nat Genet 2001, 27:48-54. 
4. Breyne P, Dreesen R, Vandepoele K, De Veylder L, Van Breusegem F, Callewaert L, Rombauts S, Raes J, Cannoot B, Engler G, et al.: Transcriptome analysis during cell division in plants. Proc Natl Acad Sci USA 2002, 99:14825-14830.

5. Menges M, Hennig L, Gruissem W, Murray JA: Genome-wide gene expression in an Arabidopsis cell suspension. Plant Mol Biol 2003, 53:423-442.

6. Simon I, Barnett J, Hannett N, Harbison CT, Rinaldi NJ, Volkert TL, Wyrick JJ, Zeitlinger J, Gifford DK, Jaakkola TS, et al.: Serial regulation of transcriptional regulators in the yeast cell cycle. Cell 2001, 106:697-708.

7. Muller GA, Engeland K: The central role of CDE/CHR promoter elements in the regulation of cell cycle-dependent gene transcription. FEBS $J$ 2010, 277:877-893.

8. Berckmans B, De Veylder L: Transcriptional control of the cell cycle. Curr Opin Plant Biol 2009, 12:599-605.

9. van den Heuvel S, Dyson NJ: Conserved functions of the pRB and E2F families. Nat Rev Mol Cell Biol 2008, 9:713-724.

*10. Chen D, Pacal M, Wenzel P, Knoepfler PS, Leone G, Bremner R: Division and apoptosis of E2f-deficient retinal progenitors. Nature 2009, 462:925-929.

This paper shows by using conditional genetic knockout in the mouse retina that all three activating E2fs are dispensable for normal division but required for cell survival.

*11. Wang S, Gu Y, Zebell SG, Anderson LK, Wang W, Mohan R, Dong X: A noncanonical role for the CKI-RB-E2F cell-cycle signaling pathway in plant effector-triggered immunity. Cell Host Microbe 2014, 16:787-794.

This paper reports on a triple $e 2 f a ; e 2 f b ; 2 f c$ genetic knockout mutant that is surprisingly normal but comprimised in effector triggered immunity related to the induction of cell death during the hypersensitive reaction.

12. Ito $\mathrm{M}$ : Conservation and diversification of three-repeat Myb transcription factors in plants. $J$ Plant Res 2005, 118:61-69.

13. Gonzalez N, Vanhaeren H, Inze D: Leaf size control: complex coordination of cell division and expansion. Trends Plant Sci 2012, 17:332-340.

**14. Kobayashi K, Suzuki T, Iwata E, Nakamichi N, Chen P, Ohtani M, Ishida T, Hosoya H, Muller S, Leviczky T, et al.: Transcriptional repression by MYB3R proteins regulates plant organ growth. EMBO J 2015, 34:1992-2007. 
The authors identified three MYB3R proteins, MYB3R1/3/5, as transcriptional repressor, that maintain the late cell cycle genes repressed outside of the G2/M in the cell cycle, and upon and after cells exit proliferation during organ development. Repressor MYB3R3 and activator MYB3R4 separately constitute multi-protein complexes together with RBR and specific E2F isoforms (E2FC and E2FB), which probably have distinct functions.

*15. Cruz-Ramirez A, Diaz-Trivino S, Wachsman G, Du Y, Arteaga-Vazquez M, Zhang $\mathrm{H}$, Benjamins R, Blilou I, Neef AB, Chandler V, et al.: A SCARECROW-RETINOBLASTOMA protein network controls protective quiescence in the Arabidopsis root stem cell organizer. PLoS Biol 2013, 11:e1001724.

This paper shows the requirement of RBR to maintain quiescence.

16. Borghi L, Gutzat R, Futterer J, Laizet Y, Hennig L, Gruissem W: Arabidopsis RETINOBLASTOMA-RELATED is required for stem cell maintenance, cell differentiation, and lateral organ production. Plant Cell 2010, 22:1792-1811.

*17. Sadasivam S, DeCaprio JA: The DREAM complex: master coordinator of cell cycle-dependent gene expression. Nat Rev Cancer 2013, 13:585-595.

This is an excellent recent review paper on the DREAM complex in animals.

18. Lipsick JS: synMuv verite--Myb comes into focus. Genes Dev 2004, 18:2837-2844.

19. Latorre I, Chesney MA, Garrigues JM, Stempor P, Appert A, Francesconi M, Strome S, Ahringer J: The DREAM complex promotes gene body H2A.Z for target repression. Genes Dev 2015, 29:495-500.

*20. Guiley KZ, Liban TJ, Felthousen JG, Ramanan P, Litovchick L, Rubin SM: Structural mechanisms of DREAM complex assembly and regulation. Genes Dev 2015, 29:961-974.

In human, assembly and disassembly of DREAM complex rely on the phosphorylation of component proteins, LIN52 and Rb-related protein p130 by DYRK1A kinase that promotes cellular quiescence and CDK4/6 important for G1 phase progression, respectively

21. Fischer M, DeCaprio JA: Does Arabidopsis thaliana DREAM of cell cycle control? EMBO J 2015, 34:1987-1989.

22. Harashima $\mathrm{H}$, Sugimoto $\mathrm{K}$ : Integration of developmental and environmental 
signals into cell proliferation and differentiation through RETINOBLASTOMA-RELATED 1. Curr Opin Plant Biol 2016, 29:95-103.

23. Chen HZ, Tsai SY, Leone G: Emerging roles of E2Fs in cancer: an exit from cell cycle control. Nat Rev Cancer 2009, 9:785-797.

24. del Pozo JC, Boniotti MB, Gutierrez C: Arabidopsis E2Fc functions in cell division and is degraded by the ubiquitin-SCF(AtSKP2) pathway in response to light. Plant Cell 2002, 14:3057-3071.

25. del Pozo JC, Diaz-Trivino S, Cisneros N, Gutierrez C: The balance between cell division and endoreplication depends on E2FC-DPB, transcription factors regulated by the ubiquitin-SCFSKP2A pathway in Arabidopsis. Plant Cell 2006, 18:2224-2235.

*26. Gutzat R, Borghi L, Futterer J, Bischof S, Laizet Y, Hennig L, Feil R, Lunn J, Gruissem W: RETINOBLASTOMA-RELATED PROTEIN controls the transition to autotrophic plant development. Development 2011, 138:2977-2986.

The authors report on an RBR cosupression line that shows abrogated developmental transition during seedling establishment leading to tumourous growth in plants.

27. De Veylder L, Beeckman T, Beemster GT, de Almeida Engler J, Ormenese S, Maes S, Naudts M, Van Der Schueren E, Jacqmard A, Engler G, et al.: Control of proliferation, endoreduplication and differentiation by the Arabidopsis E2Fa-DPa transcription factor. EMBO J 2002, 21:1360-1368.

*28. Magyar Z, Horvath B, Khan S, Mohammed B, Henriques R, De Veylder L, Bako L, Scheres B, Bogre L: Arabidopsis E2FA stimulates proliferation and endocycle separately through RBR-bound and RBR-free complexes. EMBO J 2012, 31:1480-1493.

The authors discoverred that E2FA in complex with RBR functions as a repressor for DNA replication and of differentiation.

29. Magyar Z, De Veylder L, Atanassova A, Bako L, Inze D, Bogre L: The role of the Arabidopsis E2FB transcription factor in regulating auxin-dependent cell division. Plant Cell 2005, 17:2527-2541.

30. Heyman J, Van den Daele H, De Wit K, Boudolf V, Berckmans B, Verkest A, Alvim Kamei CL, De Jaeger G, Koncz C, De Veylder L: Arabidopsis ULTRAVIOLET-B-INSENSITIVE4 maintains cell division activity by temporal inhibition of the anaphase-promoting complex/cyclosome. Plant Cell 
2011, 23:4394-4410.

31. Wenzel PL, Chong JL, Saenz-Robles MT, Ferrey A, Hagan JP, Gomez YM, Rajmohan R, Sharma N, Chen HZ, Pipas JM, et al.: Cell proliferation in the absence of E2F1-3. Dev Biol 2011, 351:35-45.

32. Haga N, Kobayashi K, Suzuki T, Maeo K, Kubo M, Ohtani M, Mitsuda N, Demura T, Nakamura K, Jurgens G, et al.: Mutations in MYB3R1 and MYB3R4 cause pleiotropic developmental defects and preferential down-regulation of multiple G2/M-specific genes in Arabidopsis. Plant Physiol 2011, 157:706-717.

33. Ito M, Araki S, Matsunaga S, Itoh T, Nishihama R, Machida Y, Doonan JH, Watanabe A: G2/M-phase-specific transcription during the plant cell cycle is mediated by c-Myb-like transcription factors. Plant Cell 2001, 13:1891-1905.

34. Ito M, Iwase M, Kodama H, Lavisse P, Komamine A, Nishihama R, Machida Y, Watanabe A: A novel cis-acting element in promoters of plant B-type cyclin genes activates M phase-specific transcription. Plant Cell 1998, 10:331-341.

35. Haga N, Kato K, Murase M, Araki S, Kubo M, Demura T, Suzuki K, Muller I, Voss U, Jurgens G, et al.: R1R2R3-Myb proteins positively regulate cytokinesis through activation of KNOLLE transcription in Arabidopsis thaliana. Development 2007, 134:1101-1110.

36. Araki S, Ito M, Soyano T, Nishihama R, Machida Y: Mitotic cyclins stimulate the activity of c-Myb-like factors for transactivation of G2/M phase-specific genes in tobacco. $J$ Biol Chem 2004, 279:32979-32988.

37. Kato K, Galis I, Suzuki S, Araki S, Demura T, Criqui MC, Potuschak T, Genschik P, Fukuda H, Matsuoka K, et al.: Preferential up-regulation of G2/M phase-specific genes by overexpression of the hyperactive form of NtmybA2 lacking its negative regulation domain in tobacco BY-2 cells. Plant Physiol 2009, 149:1945-1957.

38. Wyrzykowska J, Schorderet M, Pien S, Gruissem W, Fleming AJ: Induction of differentiation in the shoot apical meristem by transient overexpression of a retinoblastoma-related protein. Plant Physiol 2006, 141:1338-1348.

39. Korenjak M, Taylor-Harding B, Binne UK, Satterlee JS, Stevaux O, Aasland R, White-Cooper H, Dyson N, Brehm A: Native E2F/RBF complexes contain Myb-interacting proteins and repress transcription of developmentally controlled E2F target genes. Cell 2004, 119:181-193. 
40. Mehdi S, Derkacheva M, Ramstrom M, Kralemann L, Bergquist J, Hennig L: The WD40 Domain Protein MSI1 Functions in a Histone Deacetylase Complex to Fine-Tune Abscisic Acid Signaling. Plant Cell 2016, 28:42-54.

41. Jullien PE, Mosquna A, Ingouff M, Sakata T, Ohad N, Berger F: Retinoblastoma and its binding partner MSI1 control imprinting in Arabidopsis. PLoS Biol 2008, 6:e194.

42. Sadasivam S, Duan S, DeCaprio JA: The MuvB complex sequentially recruits B-Myb and FoxM1 to promote mitotic gene expression. Genes Dev 2012, 26:474-489.

43. Olson BJ, Oberholzer M, Li Y, Zones JM, Kohli HS, Bisova K, Fang SC, Meisenhelder J, Hunter T, Umen JG: Regulation of the Chlamydomonas cell cycle by a stable, chromatin-associated retinoblastoma tumor suppressor complex. Plant Cell 2010, 22:3331-3347.

*44. Narasimha AM, Kaulich M, Shapiro GS, Choi YJ, Sicinski P, Dowdy SF: Cyclin D activates the Rb tumor suppressor by mono-phosphorylation. Elife 2014, 3.

The authors convincingly show that during the transition through G1, Rb become phosphorylated on alternative individual sites, leading to mono-phosphorylated $\mathrm{Rb}$ forms with distinct properties; such as the association with activator E2F1 to regulate checkpoint arrest and apoptosis during DNA damage, or the dissociation from repressor E2F4 to enable the entry into G1/S.

45. Popescu SC, Popescu GV, Bachan S, Zhang Z, Gerstein M, Snyder M, Dinesh-Kumar SP: MAPK target networks in Arabidopsis thaliana revealed using functional protein microarrays. Genes Dev 2009, 23:80-92.

46. Lopez-Bucio JS, Dubrovsky JG, Raya-Gonzalez J, Ugartechea-Chirino Y, Lopez-Bucio J, de Luna-Valdez LA, Ramos-Vega M, Leon P, Guevara-Garcia AA: Arabidopsis thaliana mitogen-activated protein kinase 6 is involved in seed formation and modulation of primary and lateral root development. $J$ Exp Bot 2014, 65:169-183.

47. Ikeuchi M, Sugimoto K, Iwase A: Plant callus: mechanisms of induction and repression. Plant Cell 2013, 25:3159-3173.

48. Wen H, Andrejka L, Ashton J, Karess R, Lipsick JS: Epigenetic regulation of gene expression by Drosophila Myb and E2F2-RBF via the Myb-MuvB/dREAM complex. Genes Dev 2008, 22:601-614. 
49. Wildermuth MC: Modulation of host nuclear ploidy: a common plant biotroph mechanism. Curr Opin Plant Biol 2010, 13:449-458.

50. Chandran D, Inada N, Hather G, Kleindt CK, Wildermuth MC: Laser microdissection of Arabidopsis cells at the powdery mildew infection site reveals site-specific processes and regulators. Proc Natl Acad Sci USA 2010, 107:460-465.

51. Eichmann R, Schäfer P: Growth versus immunity--a redirection of the cell cycle? Curr Opin Plant Biol 2015, 26:106-112.

52. Hamdoun S, Zhang C, Gill M, Kumar N, Churchman M, Larkin JC, Kwon A, Lu H: Differential Roles of Two Homologous Cyclin-Dependent Kinase Inhibitor Genes in Regulating Cell Cycle and Innate Immunity in Arabidopsis. Plant Physiol. 2016, 170: 515-527.

53. Vlieghe K, Boudolf V, Beemster GT, Maes S, Magyar Z, Atanassova A, de Almeida Engler J, De Groodt R, Inzé D, De Veylder L: The DP-E2F-like gene DEL1 controls the endocycle in Arabidopsis thaliana. Curr Biol 2005, 15:59-63.

54. Litovchick L, Sadasivam S, Florens L, Zhu XP, Swanson SK, Velmurugan S, Chen RS, Washburn MP, Liu XS, DeCaprio JA: Evolutionarily conserved multisubunit RBL2/p130 and E2F4 protein complex represses human cell cycle-dependent genes in quiescence. Molecular Cell 2007, 26:539-551.

55. Harrison MM, Ceol CJ, Lu X, Horvitz HR: Some C. elegans class B synthetic multivulva proteins encode a conserved LIN-35 Rb-containing complex distinct from a NuRD-like complex. Proc Natl Acad Sci USA 2006, 103: 1678216787.

56. Schmit F, Korenjak M, Mannefeld M, Schmitt K, Franke C, von Eyss B, Gagrica S, Hänel F, Brehm A, Gaubatz S: LINC, a human complex that is related to pRB-containing complexes in invertebrates regulates the expression of G2/M genes. Cell Cycle 2007, 6:1903-1913.

57. Lewis PW, Beall EL, Fleischer TC, Georlette D, Link AJ, Botchan MR: Identification of a Drosophila Myb-E2F2/RBF transcriptional repressor complex. Genes Dev 2004, 18:2929-2940. 


\section{Figure legends}

\section{Figure 1.}

Schematic model for transcriptional regulation of the late cell cycle genes by activator- and repressor-type MYB3Rs in Arabidopsis.

In proliferating cells, the repressor-type MYB3Rs are responsible for keeping the late cell cycle genes suppressed outside of G2/M interval, until the transcriptional repression is lost concomitant with the activation of activator type-MYB3Rs. This switch-like transition triggers amplification of activator-type MYB3Rs through positive feedback loop mechanisms at transcriptional and post-transcriptional levels to enable the burst of target gene expression and irreversible entry into mitosis. In differentiated cells, the late cell cycle genes are stably maintained in a long-term silenced state by the function of repressor-type MYB3Rs.

\section{Figure 2.}

\section{Regulation of cell cycle and quiescence by DREAM-like complexes in plants}

In proliferating cells, the early cell cycle genes are repressed by the E2FB-DPA/B-RBR complex during G1. Phosphorylation of RBR by CYCD-CDKA;1 induces the release of active E2F-DPA/B to induce the transcription of early cell cycle genes. Later, E2FC may have a role in repressing transcription of the early cell cycle genes. This repression may be also accompanied by the action of DEL transcription factors, known as atypical E2Fs, which act as monomer in an RBR and DP-independent manner [53]. During G1, S and early G2, the late cell cycle genes are transcriptionally suppressed by the protein complex containing repressor-type MYB3R, RBR and possibly MuvB-like core complex. During late G2, this repressive complex on the target promoters is replaced by the MYB3R-E2F activator complex (containing activator-type MYB3R, E2FB, RBR, and MuvB-like core complex), triggering its auto-activation through the positive feedback loops and allowing transcription of the late cell cycle genes. In post-mitotic cells, the late cell cycle genes are kept in silenced state by the separate MYB3R-E2F complex with a repressive function (containing repressor-type MYB3R, E2FC, RBR and MuvB-like complex).

\section{Box 1. DREAM complex}


The DREAM (DP, Rb-like, E2F, and MuvB) complex is known as a key regulator to achieve and maintain cellular quiescence by repressing cell cycle-regulated genes (both early and late cell cycle genes) in mammalian cells [54]. The DREAM consists of an Rb-related tumor suppressor family protein (p130 or p107), E2F family protein (E2F4 or E2F5) and its dimerizing partner DP, and a five-component MuvB core complex (LIN9, LIN37, LIN52, LIN54, and RBBP4). Interestingly, these DREAM components overlap with genetically-defined synthetic multivulval class B (MuvB) proteins in nematode [39], and shared with the evolutionarily conserved complexes that are biochemically identified from fly (dREAM) [18,39] and nematode (DRM) [55]. It should be noted that some of these complexes have synonyms when they were identified independently by the different groups, such as LINC (LIN complex) in human [56] and MMB (Myb-MuvB) in fruit fly [57], which are equivalent to DREAM and dREAM, respectively. The biochemical functions of these proteins are not known, with the exception of RBAP4 and LIN54, which have the abilities to bind histones and DNA, respectively. The mechanism of DREAM-mediated transcriptional regulation has not been well understood, but it is clear that its core complex recruits key cell cycle transcription factors, including E2F4/5, B-Myb, and FoxM1. In some cases, the complex are co-purified with chromatin regulators such as histone deacetylases and L(3)mbt that binds modified histones for chromatin compaction [51], suggesting transcriptional regulation through chromatin status. 
Proliferating cells

(meristem, developing organs)
Differentiated cells (fully developed organs)

\section{Transcription of}

G2/M genes

Switching from Rep-Myb to Act Myb
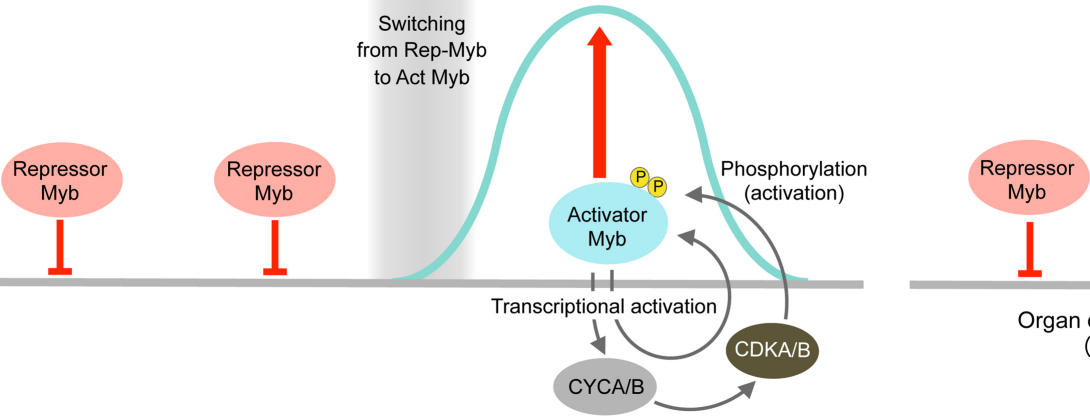

Organ development (Time) 
Table 1 Components of DREAM or related complex

\begin{tabular}{lllll}
\hline $\begin{array}{c}\text { Human } \\
\text { (DREAM) }\end{array}$ & $\begin{array}{c}\text { Fruit fly } \\
\text { (dREAM/MMB) }\end{array}$ & \multicolumn{2}{c}{$\begin{array}{c}\text { Arabidopsis } \\
\text { (DREAM-like?) }\end{array}$} & \\
\hline B-Myb & Activation? & Repression? & Function \\
\hline E2F4/5 & E2F2 & E2FB & E2FC & Forming dimer with DP to bind DNA \\
\hline DP1/2/3 & Dp & DPA & DPA? & Forming dimer with E2F to bind DNA \\
p107, p130 & Rbf1, Rbf2 & RBR & RBR & Binding to E2F to inhibit transcription \\
\hline LIN9 & Mip130 & ALY3 & ALY2, ALY3 & Binding to DNA with A/T-rich sequence \\
\hline LIN54 & Mip120 & TCX5 & TCX5 & Sequence-specific DNA binding \\
\hline RBBP4 & Caf1 (p55) & MSI? & MSI? & Binding to chromatin \\
\hline LIN37 & Mip40 & No homolog in plants & Unknown \\
\hline LIN52 & dLIN52 & No homolog in plants & Interacting with p107/p130 \\
\hline FoxM1 & $?$ & No homolog in plants & Sequence-specific DNA binding \\
& L(3)mbt & No homolog in plants & Binding to methylated histone \\
\hline HDAC3 & Rpd3 & HAD? & HAD? & Histone acetylase \\
\hline
\end{tabular}

